\title{
Inguinale Lymphadenopathie bei heterosexuellem Patienten
}

\section{Inguinal Lymphadenopathy in a Heterosexual Patient Without Risk Factors for Sexualy Transmitted Disease}

Autoren

Institute

\author{
D. Schneider ${ }^{1}$, J. Gubler ${ }^{1}$, D. Ochsner ${ }^{2}$, M. Fliegner
}

Medizinische Klinik, Stadtspital Triemli Zürich/Schweiz (Chefarzt: Prof. Dr. O. Oelz)

Institut für Pathologie, Stadtspital Triemli Zürich/Schweiz (Chefarzt: Prof. Dr. R. Maurer)

\section{Bibliografie}

DOI $10.1055 / \mathrm{s}-2006-944586$ Akt Dermatol 2006; 32; 421-424 @ Georg Thieme Verlag KG Stuttgart · New York ISSN 0340-2541

Korrespondenzadresse Dr. Markus Fliegner Medizinische Klinik Stadtspital Triemli Zürich Birmensdorferstrasse 497 8063 Zürich/Schweiz markus.fliegner@triemli.stzh.ch

\section{Zusammenfassung $\nabla$}

Ein 44-jähriger Mann, der keine Reisen in Endemiegebiete unternommen hatte, in einer stabilen, langjährigen heterosexuellen Beziehung lebt und homosexuelle Kontakte auch auf Nachfrage verneint, stellt sich mit einem beidseitigen „groove sign“ vor. Ursächlich ist ein Lymphogranuloma venereum (LGV) vom Serotyp L2b, dem Typus, das aktuell im Zusammenhang mit einer ulzerativen Proktitis bei MSM (Men having Sex with Men) beschrieben wird. Bei aller Unsicherheit hinsichtlich anamnestischer Angaben zum

\section{Einleitung}

\section{$\nabla$}

Das Lymphogranuloma venereum als sexuell übertragbare Erkrankung erlebt aktuell durch das Auftreten im Zusammenhang mit einer Proktitis bei homosexuellen Patienten durch den Genotyp L2b (Amsterdam) eine vermehrte Aufmerksamkeit. Wir berichten über den Fall eines unerwarteten Nachweises des LGV-Genotyps Amsterdam bei einem heterosexuellen Patienten.

\section{Kasuistik \\ $\nabla$ \\ Anamnese}

Der 44-jährige Architekt bemerkte 10 Tage zuvor Lymphknotenschwellungen inguinal rechts und später auch links, die zunahmen und druckschmerzhaft wurden. Der Patient hatte weder Fieber noch andere systemische Krankheitszeichen. Er hatte keine Auslandsreisen nach Afrika, Asien, Südamerika oder in die Karibik unternommen und hielt keine Haustiere. Er lebte in einer langjährigen, stabilen, heterosexuellen Beziehung; in den letzten 12 Monaten habe er keinen ungeschützten Geschlechtsverkehr mit anderen
Sexualverhalten zeigt der Fall, dass an ein LGV auch bei Patienten außerhalb von Risikogruppen gedacht werden muss. Die Präsentation kann sowohl den klassischen Verlauf mit einer Lymphadenopathie („groove sign“) nehmen als auch sich insbesondere bei MSM nach ungeschütztem anorezeptivem Verkehr mit einer Proktitis manifestieren. Die vorliegende Kasuistik zeigt, dass der bislang nur bei MSM im Rahmen einer Proktitis beschriebene Serotyp LGV L2b möglicherweise auch auf heterosexuellem Weg übertragen werden und den klassischen Verlauf des LGV mit inguinaler Lymphadenopathie nehmen kann.

Partnerinnen gehabt und Analverkehr oder homosexuelle Kontakte wurden explizit verneint.

\section{Aufnahmebefund}

Afebriler Patient in gutem Allgemeinzustand; zwei $10 \times 4 \mathrm{~cm}$ bzw. $6 \times 3 \mathrm{~cm}$ große, erhabene, derbe, gerötete und überwärmte Lymphknotenpakete inguinal beidseits. Die übrigen Lymphknotenstationen waren ebenso wie der körperliche Untersuchungsbefund (incl. Genitalinspektion und rektaler Untersuchung) unauffällig.

\section{Befunde diagnostischer Untersuchungen $\nabla$}

Routinelabor

CRP $9.3 \mathrm{mg} / \mathrm{L} \quad(<10)$, Leukozyten $7.4 \times 10^{9} / \mathrm{L}$ (3.7-9.6), davon $31.5 \%$ stabkernige sowie einige stark reaktive Lymphozyten und Monozyten mit stark gelappten Kernen. Die Routineparameter inkl. Urin-Status waren unauffällig.

\section{Serologie}

Serologien auf Toxoplasmose, HIV, Lues und Gonorrhö zeigten keine durchgemachte oder frische Infektion; für EBV fand sich eine Seronarbe. 
Tab. 1 Chlamydien-Serologie und deren Laborinterpretation: genus-spezifische Komplementfixation (KBR) und spezies-spezifische Immunfluoreszenz (IF); pathologische Titer sind in Fettdruck hervorgehoben

\begin{tabular}{|c|c|c|c|c|}
\hline \multirow{2}{*}{$\begin{array}{l}\text { Parameter } \\
\text { Chlamydia spp. } \\
\text { (KBR) }\end{array}$} & \multicolumn{3}{|c|}{ Ergebnis } & \multirow{3}{*}{$\begin{array}{l}\text { Interpretation } \\
\text { Infektion mit } \\
\text { Chlamydien- } \\
\text { Spezies }\end{array}$} \\
\hline & \multicolumn{3}{|l|}{$1: 40$} & \\
\hline & & & & \\
\hline C. psittaci & $<1 \cdot 10$ & $1 \cdot 10$ & $1: 80$ & \\
\hline (IF) & & & & Infektion \\
\hline $\begin{array}{l}\text { C. pneumoniae } \\
\text { (IF) }\end{array}$ & $1: 40$ & $1: 10$ & $1: 320$ & $\begin{array}{l}\text { durchgemachte } \\
\text { Infektion }\end{array}$ \\
\hline $\begin{array}{l}\text { C. trachomatis } \\
\text { (IF) }\end{array}$ & $<1: 10$ & $1: 20$ & $1: 20$ & $\begin{array}{l}\text { beginnende } \\
\text { Infektion? }\end{array}$ \\
\hline
\end{tabular}

- Tab. 1 zeigt die Ergebnisse der Chlamydien-Serologie.

\section{Histologie}

Lymphadenitis mit herdförmigen, teils landkartenartigen Abszessen mit zentralen granulozytären Infiltraten und randständigem Histiozytensaum, teils mit epitheloidzelligen Histiozyten (৫ Abb. 1).

\section{Molekulardiagnostik}

Aus der Lymphknotenbiopsie PCR auf Bartonella Spezies negativ; Spezies-spezifische PCR auf Chlamydia trachomatis positiv, Genotypisierung (PCR-Amplifikation des omp1-Gens und Sequenzierung) ergibt LGV Genotyp L2b.

\section{Therapie und Verlauf}

$\nabla$

Zum Ausschluss eines rasch wachsenden Lymphoms sowie zur Gewebegewinnung für eine molekulardiagnostische Methode ließen wir eine Lymphknotenexzision durchführen. Anhand von Klinik, Serologie, Histologie und PCR stellten wir die Diagnose eines Lymphogranuloma venereum L2b und begannen eine Therapie mit Doxycyclin $2 \times 100 \mathrm{mg}$ für drei Wochen. Nach fünf Tagen kam es darunter zu einer Einschmelzung des Befundes in der rechten Leiste, weshalb eine operative Abszessabdeckelung nötig wurde. Nach einmaliger Entleerung von $70 \mathrm{ml}$ Pus war der weitere Verlauf unkompliziert. Eine Bakteriologie blieb negativ.

Die genauere Diagnostik des Übertragungsmodus durch Untersuchung der asymptomatischen Partnerin war leider nicht möglich, da diese bereits eine ambulant veranlasste Antibiose erhalten hatte. Wir empfahlen diese auf Doxycyclin $2 \times 100 \mathrm{mg}$ für 7 Tage zu ändern. Der Patient verneinte auch nach ausführlicher Besprechung der Diagnose homosexuelle Kontakte oder ungeschützten Geschlechtsverkehr außerhalb seiner Beziehung.

\section{Diskussion}

$\nabla$

Ein Lymphogranuloma venereum, verursacht durch das gramnegative, sich obligat intrazellulär vermehrende Bakterium C. trachomatis mit einem der drei Serovare L1, L2 und L3, war bis vor kurzem eine in den Industrieländern nur sporadisch beschriebene Geschlechtserkrankung. Das LGV galt als in Afrika, Asien, Südamerika oder der Karibik endemisch ( $\bullet$ Abb. 2).

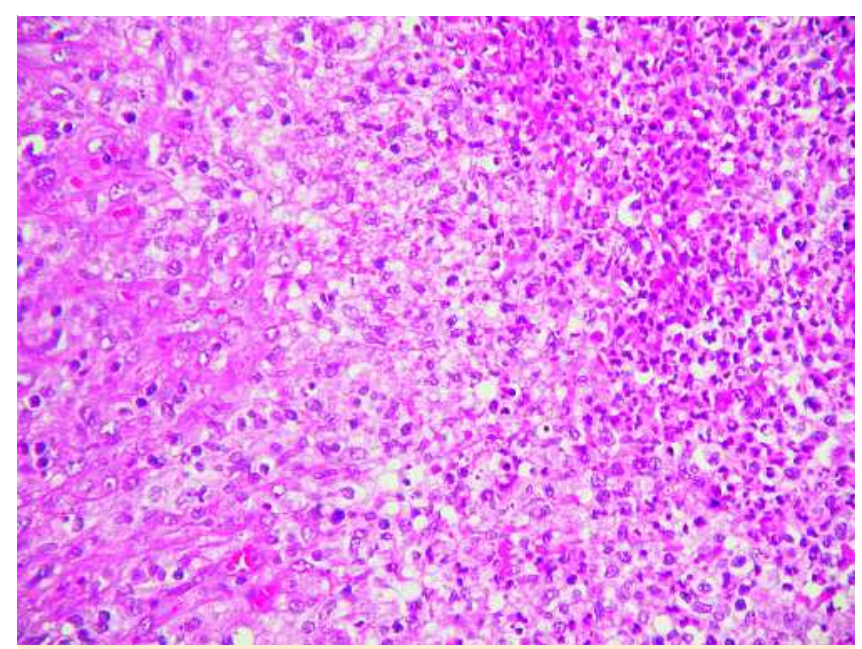

Abb. 1 Detailaufnahme mit Anteilen der zentral abszedierenden Entzündung und des palisadierenden Histiozytensaumes.

Im Primärstadium entsteht nach einer Inkubationszeit von 3-30 Tagen am Ort der Inokulation ein nur wenige Tage bestehendes, unbehandelt abheilendes Ulkus. Eine Primärläsion in Harnröhre, Vagina oder Rektum bleibt oft unbemerkt [1].

Nach zwei bis sechs Wochen kommt es zu einem Sekundärstadium mit schmerzhafter Lymphadenopathie: je nach Infektionsort und lymphatischem Abfluss können diese Lymphknoten inguinal (typisches „groove sign“ durch Vergrößerung der durch das Poupart'sche Ligament getrennten femoralen und inguinalen Lymphknoten) ( $\triangle$ Abb.3), retroperitoneal (bei Frauen oder bei anorezeptivem Geschlechtsverkehr), zervikal (bei Oralverkehr) oder auch mediastinal/supraklavikulär (z. B. bei akzidenteller Inhalation der Erreger durch Laborpersonal) liegen. Beim anorektalen Syndrom nach anorezeptivem Geschlechtsverkehr kommt es zu einer hämorrhagischen Proktitis und Hyperplasie des intestinalen und perirektalen Lymphgewebes. Neben einer akuten Verlaufsform mit massenhafter Bildung von Elementarkörperchen, Zellwandlyse und rascher Erregerausbreitung kann es auch zu einer persistierenden Infektion mit Ausschleusung von infektiösen Elementarkörperchen durch die intakt bleibende Zellwand mittels Exozytose und chronisch rezidivierenden Krankheitsschüben kommen. Die mantelartige Umlagerung des Zellkerns durch Einschlusskörperchen hat den Erregern ihren Namen gegeben („chlamys“, griech. der Mantel).

Das seltener vorkommende Tertiärstadium ist gekennzeichnet durch eine Fibrose mit lymphatischer Abflussstörung, genitaler Elephantiasis oder Esthiomene (Infektion der lymphatischen Gefäße von Skrotum und Penis oder Vulva) [2].

Seit 2002 werden Ausbrüche einer LGV-Proktitis unter homosexuellen, meist HIV-positiven Patienten, verursacht durch den Serotyp L2b (Amsterdam) in verschiedenen westlichen Ländern wie Belgien, den Niederlanden, Großbritannien, Frankreich, Deutschland, USA und der Schweiz beschrieben [3,11]. LGV L2b konnte retrospektiv erstmalig in Proben aus den 80er-Jahren in San Francisco nachgewiesen werden. Ob die Inzidenz aktuell wirklich zunimmt oder nur Aufmerksamkeit und Diagnostik verbessert sind, wird diskutiert [10].

Die Differenzialdiagnose des LGV umfasst im Stadium des genitalen Ulkus den weichen Schanker, Herpes, Syphilis und die Donovaniose. Im Stadium der Lymphadenopathie kommen HIV, EBV, CMV, Bartonellose und maligne Lymphome hinzu. Je nach 


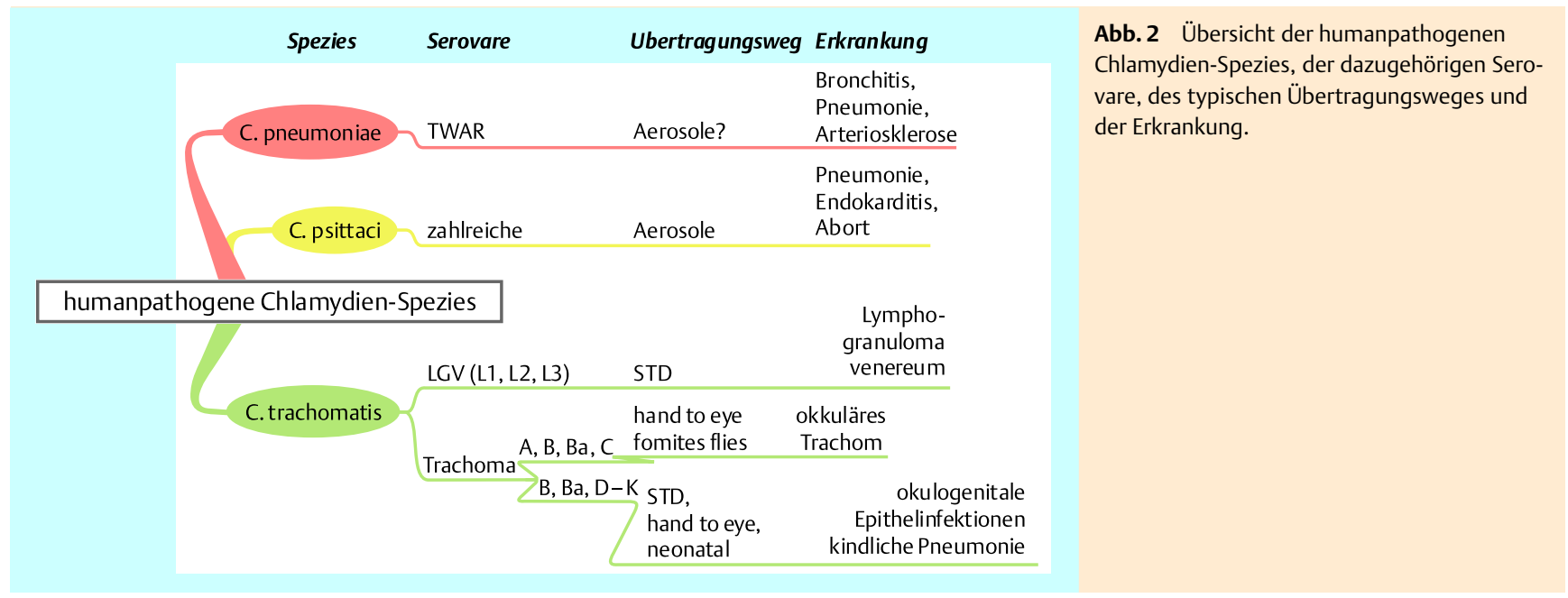

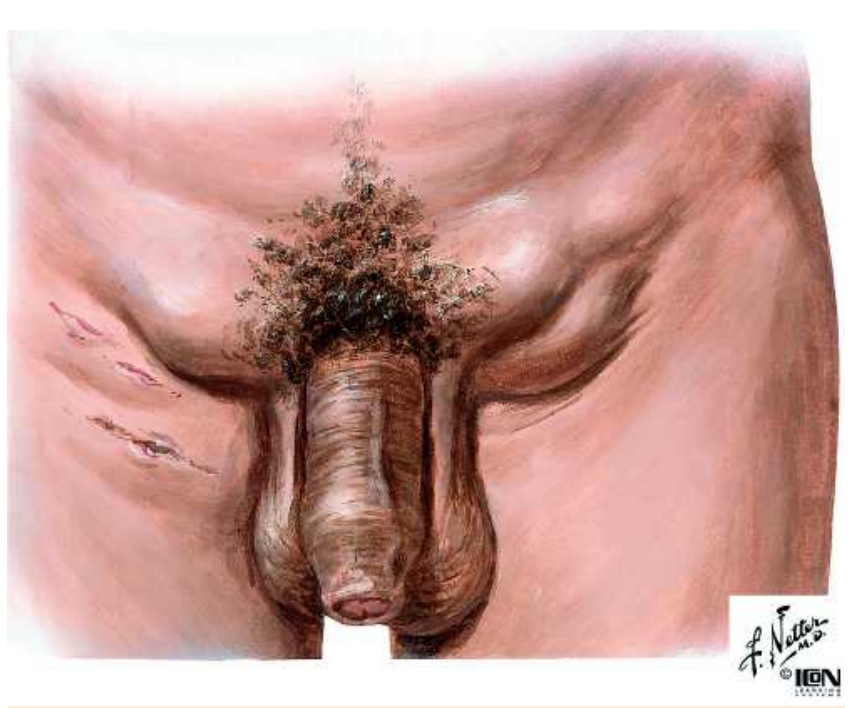

Abb. 3 Sekundärstadium mit inguinaler Lymphadenopathie (Abdruck mit Genehmigung des Elsevier-Verlages (www.netterimages.com).

endemischer Situation ist an eine Lymphknotentuberkulose oder auch an die Pest zu denken.

Die Diagnosestellung kann serologisch unterstützt werden, sollte aber heute molekulardiagnostisch aus Läsionen erfolgen. Der kulturelle Nachweis von Erregern aus klinischen Läsionen ist kostspielig und die Sensitivität dieser Methode gering. Die Komplementfixation ist lediglich genus-spezifisch und nicht in der Lage, zwischen C. trachomatis, C. psittaci oder C. pneumoniae zu differenzieren. Das invasivere LGV mit den Serovaren L1, L2 und L3 soll dabei zu höheren Antikörpertitern als unkomplizierte genitale Infektionen mit $C$. trachomatis der Serovare D-K führen. Ein Titer von $>1: 256$ gilt als suggestiv für, ein Titer von $<1: 32$ als nahezu ausschließend für eine LGV-Infektion [6]. Die Immunfluoreszenzmikroskopie wäre in der Lage, Infektionen der einzelnen Chlamydienspezies zu differenzieren, ist in der Praxis aber selten verfügbar. Die Verlässlichkeit von Enzymimmunoassays ist unklar [4]. Die sensitivste und hochspezifische Methode zum LGV-Erregernachweis ist eine DNA-Amplifikation aus Bubomaterial (Aspirat oder Exzidat). Für rektale und pharyngeale Proben ist diese Methode aktuell noch nicht lizensiert. Bei einem positiven Test-Ergebnis sollte eine Kontrolle durch einen PCRTest eines anderen Herstellers erfolgen und bei Bestätigung eine Genotypisierung durchgeführt werden [1], die gegenwärtig nur in wenigen Speziallaboratorien zur Verfügung steht. Die Histologie zeigt eine lymphoproliferative Reaktion und Nekrosezonen in den Lymphknoten.

Zur Therapie der bubonic form und der anogenitalen Form des LGV wird Doxycyclin $2 \times 100 \mathrm{mg} / \mathrm{d}$ für 21 Tage empfohlen, 7 Tage länger also, als für Chlamydieninfektionen der Serovare B-K [3]. Eine Einmaltherapie mit Azithromycin $1 \mathrm{~g}$ [9] ist nicht verlässlich [5]. Kleinere Abszedierungen können evt. über eine laterale Aspiration durch gesunde Haut drainiert [8], größere müssen operativ saniert werden. In der Schwangerschaft wird Erythromycin als Alternative empfohlen. Wie bei anderen STD ist die Beratung und Behandlung der Kontaktpersonen nötig; asymptomatische Partner sollten entweder nachkontrolliert oder mit Doxycyclin $2 \times 100 \mathrm{mg}$ für 7 Tage behandelt werden. Bemerkenswert am vorliegenden Fall ist, bei aller Einschränkung von anamnestischen Angaben zum Sexualverhalten, die vermutliche Übertragung eines LGV L2b durch einen heterosexuellen Kontakt ohne das Vorliegen der bislang beschriebenen üblichen Risikofaktoren wie HIV-Positivität, Promiskuität, Analverkehr, geringes Bildungsniveau, Anamnese für STD oder Akquisition in einem Endemiegebiet [7]. LGV L2b scheint dabei eine klassische Verlaufsform der LGV zu verursachen. Die Ergebnisse der extern veranlassten Chlamydien-Serologien (Laborinterpretation als „Kontakt mit C. psittaci, chronische Infektion mit C. pneumoniae sowie evt. akute Infektion mit C. trachomatis") illustrieren, dass eine Serologie nur mit Einschränkung für die Frage nach einem akuten LGV zu interpretieren ist.

Im November 2005 rief das Schweizerische Bundesamt für Gesundheit Ärzte bereits dazu auf, vermehrt an die Differentialdiagnose des LGV bei MSM mit den beschriebenen Symptomen der Proktitis zu denken und diese entsprechend abzuklären. Der hier beschriebene Fall verdeutlicht, dass dieses differentialdiagnostische Denken auch auf Patienten ohne übliche Risikofaktoren sowie auf eine zumindest in den Industrienationen bisher seltene klinische Manifestation erweitert werden muss.

\section{Interessenkonflikt}

Keiner der Autoren hat Verbindungen mit einer Firma, deren Produkt in dem Artikel genannt ist oder einer Firma, die ein Konkurrenzprodukt vertreibt. 


\section{Danksagung \\ $\nabla$}

Wir möchten Herrn Dr. sc. nat. D. Goldenberger, Institut für medizinische und molekulare Diagnostik AG, Zürich, für die Genotypisierung danken.

\section{Abstract}

Inguinal Lymphadenopathy in a Heterosexual Patient Without Risk Factors for Sexualy Transmitted Disease $\nabla$

A 44-year old male presented to an emergency department in Zürich with a bilateral groove sign. He is living in a longstanding heterosexual relationship, denies sexual contact with men and has not visited endemic areas of lymphogranuloma venereum. PCR identified LGV L2b, the biovar now described with the emergence of hemorrhagic proctitis in MSM (Men having Sex with Men). Since one can never completely rely on anamnestic statements of sexual behavoir, LGV must be considered in heterosexual patients, even when the usual risk factors are not present. LGV L2b may be acquired by heterosexual intercourse and may present as classic LGV with a groove sign.

\section{Literatur}

1 Lymphogranuloma-venereum-Ausbrüche bei homosexuellen Männern in Europa und Nordamerika - aktueller Stand. Epidemiologisches Bulletin Robert Koch-Institut 2005; 8: 65-66

2 Aggarwal K, Jain VK, Gupta S. Bilateral groove sign with penoscrotal elephantiasis. Sex Transm Infect 2002; 78: 458

3 Blank S, Schillinger JA, Harbatkin D. Lymphogranuloma venereum in the industrialised world. Lancet 2005; 365: 1607-1608

4 Clad A, Freidank HM, Kunze M, Schnoeckel U, Hofmeier S, Flecken U, Petersen EE. Detection of seroconversion and persistence of Chlamydia trachomatis antibodies in five different serological tests. Eur J Clin Microbiol Infect Dis 2000; 19: 932 - 937

5 Davis BT, Thiim M, Zukerberg LR. Case records of the Massachusetts General Hospital. Case 2-2006. A 31-year-old, HIV-positive man with rectal pain. N Engl J Med 2006; 354: 284-289

6 Dyck EV, Meheus A, Piot P. Laboratory diagnosis of sexually transmitted diseases. Geneva World Health Organization; 1999

7 Gotz HM, van Bergen JE, Veldhuijzen IK, Broer J, Hoebe CJ, Steyerberg EW, Coenen AJ, de Groot F, Verhooren MJ, van Schaik DT, Richardus JH. A prediction rule for selective screening of Chlamydia trachomatis infection. Sex Transm Infect 2005; 81: 24-30

8 Mabey D, Peeling RW. Lymphogranuloma venereum. Sex Transm Infect 2002; 78: 90-92

9 Nieuwenhuis RF, Ossewaarde JM, van der Meijden WI, Neumann HA. Unusual presentation of early lymphogranuloma venereum in an HIV-1 infected patient: effective treatment with $1 \mathrm{~g}$ azithromycin. Sex Transm Infect 2003; 79: $453-455$

10 Schachter J, Moncada J. Lymphogranuloma venereum: how to turn an endemic disease into an outbreak of a new disease. Start looking? Sex Transm Dis 2005; 32: $331-332$

11 Williams $D$, Churchill $D$. Ulcerative proctitis in men who have sex with men: an emerging outbreak. BMJ 2006; 332: 99-100
Buchbesprechung

Diagnostik und Förderung integrativer Kompetenz bei Psoriasis

Judith A. Bahmer

Aachen: Shaker, 2006. 245 S., 24 Abb. 29,80€.

ISBN 3-8322-5164-2

Es ist keine leichte Kost, die uns Judith Bahmer da in Form ihrer philosophischen Doktorarbeit präsentiert. Auf 245 eng beschriebenen Seiten gibt sie uns einen Einblick in Psoriasis, Psyche und ein von ihr entwickeltes sekundäres Präventionsprogramm. Doch bis man sich dahin durchgearbeitet hat braucht man Ausdauer und ein grundlegendes psychologisches Verständnis. Von der für alle Dermatologen interessanten, sehr schön zusammengefassten Geschichte und Literaturgeschichte der Psoriasis bis hin zur Psyche und Psychoneuroimmunologie liest sich das Werk sehr gut und ist ungemein informativ. Doch das sind erst 48 von 245 Seiten. Die nachfolgenden, einleitenden Kapitel sind ein Kompendium der Psychologie, das von der Theorie der Persönlichkeits-System Interaktion zu den Grenzen des entwicklungsorientierten Scannings kommt. Wer da noch folgen kann, hat bei den Kursen der psychosomatischen Grundversorgung sehr gut aufgepasst. Dann erst kommt der Hauptteil, ganz in wissenschaftlichem Stil, mit Material und Methoden, Ergebnissen und Diskussion. Dieser Teil beschreibt die eigentliche Mission dieser Arbeit, nämlich die Entwicklung eines spezifisch auf Psoriatiker ausgelegten Präventionsprogrammes, man könnte es auch Psychosomatisches Psoriasis Trainingsprogramm nennen. Die Quintessenz des Programmes ist, dass ähnlich wie bei der Neurodermitis eine spezifische Schulung von den Patienten gut aufgenommen wird, dass diese in einem solchen Programm bereitwillig ihre „Seele“ öffnen und über Probleme wie Stigmatisierung und persönliche Stressoren tatsächlich reden. Das führt zu einem signifikant verbesserten Coping mit dieser chronischen Krankheit und erstaunlicherweise langfristig auch zu einem besseren Hautzustand. Dieser Weg ist sehr mühsam und gehört in die Hände von dermatologisch interessierten Psychologen, von denen es leider nicht allzu viele gibt. Es ist für uns Dermatologen natürlich zunächst viel einfacher zu Lichttherapie oder „Biologicals“ zu greifen, um Psoriasis-Patienten zu helfen. Nach der Lektüre dieses Buches sollte es jedoch jedem klar sein, dass ein „ganzheitlicher“ Ansatz, der auch die Psychopathologie der Erkrankung berücksichtigt, dem Patienten besser gerecht wird, seine Haut schneller und nachhaltiger zur Abheilung bringt und zur Reduktion der erheblichen Komorbiditäten beitragen kann. Auch als Anleitung oder Nachschlagewerk ist dieses Buch besonders für die allenthalben sprießenden Psoriasis-Kompetenzzentren sehr zu empfehlen. Ich würde mir von Frau Bahmer eine etwas leichter lesbare, gekürzte Version wünschen, die auch psychologisch nicht ganz so versierten Dermatologen den Zugang zu diesem wichtigen Kapitel der Psoriasis erleichtert.

H. Kurzen, Mannheim 